\title{
Autonomic conditioning therapy reduces fatigue and improves global impression of change in individuals with post-acute COVID-19 syndrome
}

David Putrino ( D DAVID.PUTRINO@MOUNTSINAI.ORG)

Icahn School of Medicine at Mount Sinai

Laura Tabacof

Icahn School of Medicine at Mount Sinai https://orcid.org/0000-0002-6036-2447

Jenna Tosto-Mancuso

Icahn School of Medicine at Mount Sinai

Jamie Wood

Icahn School of Medicine at Mount Sinai

Mar Cortes

Icahn School of Medicine at Mount Sinai

Amy Kontorovich

Icahn School of Medicine at Mount Sinai

https://orcid.org/0000-0001-7400-5507

\section{Dayna McCarthy}

Icahn School of Medicine at Mount Sinai

\section{Erica Breyman}

Icahn School of Medicine at Mount Sinai https://orcid.org/0000-0002-4784-4310

\section{Leila Nasr}

Icahn School of Medicine at Mount Sinai

Adena Neglia

Icahn School of Medicine at Mount Sinai

Josh Duntz

Stasis Performance

Alex Bunt

Icahn School of Medicine at Mount Sinai

Joseph Herrera

Icahn School of Medicine at Mount Sinai

Christopher Kellner

Icahn School of Medicine at Mount Sinai 
Keywords: Post-acute COVID-19 syndrome (PACS), Autonomic Conditioning Therapy (ACT), fatigue, exercise programs

Posted Date: April 22nd, 2021

DOI: https://doi.org/10.21203/rs.3.rs-440909/v1

License: (1) This work is licensed under a Creative Commons Attribution 4.0 International License. Read Full License 


\section{Abstract}

Post-acute COVID-19 syndrome (PACS) is a collection of persistent and debilitating symptoms lasting weeks to months after acute COVID-19 infection, with fatigue most commonly reported. There is controversy surrounding the role of exercise programs for this condition, due to concerns over the potential to worsen fatigue. We developed a novel physical therapy program known as Autonomic Conditioning Therapy (ACT) for PACS, and report on the preliminary patient-reported outcome (PRO) data from individuals who completed ACT for PACS, compared with those who did not. Seventy-eight (55 [71\%] female, median [range] age 43 [12 to 78]) met the inclusion criteria and consented to have their data included in the analyses. A total of 31 (40\%) individuals completed ACT for PACS. There was withingroup improvement in fatigue in individuals who completed ACT for PACS (mean difference [95\% Cl] - 14 [-27 to -1], $p=0.03$ ), as well as greater between-group impression of change measured on the Patient Global Impression of Change scale (ACT for PACS median [range] 5 [1 to 7], no ACT for PACS 4 [1 to 7], p $<0.01)$. ACT for PACS is a novel physical therapy program that can reduce fatigue in individuals with PACS.

\section{Main Text}

Long-term symptoms associated with acute SARS-CoV-2 (COVID-19) infection may be present in up to $75 \%$ of COVID-19 survivors, ${ }^{1}$ and are not limited to those with severe acute infection or pre-existing comorbidities. $^{2-6}$ The cornerstone of post-acute COVID-19 syndrome (PACS; also referred to as "Iong COVID" or "post-COVID") is a collection of persistent and debilitating symptoms lasting weeks to months after acute COVID-19 infection in the absence of any specific organ system damage using standard clinical testing protocols. ${ }^{3,5}$ These symptoms, with fatigue being the most predominant, ${ }^{3}$ increase disability and feelings of anxiety and depression, ${ }^{6}$ and negatively impact physical function and quality of life. ${ }^{3}$ This ultimately results in reduced participation and time off work, ${ }^{2,3}$ extending the potential reach of PACS to the economy at large. This is a public health emergency, with extrapolated data suggesting that at least 2 million Americans may have or will develop PACS. ${ }^{7-9}$ This has created an urgent need for the development and validation of rehabilitation programs targeting this cohort, with the goal of reducing fatigue and disability and facilitating return to pre-COVID function.

Several dozen known symptoms of PACS have been reported. Alongside fatigue, shortness of breath, weakness, erratic heart rate and thermodysregulation are common. ${ }^{3,6}$ It has been hypothesized that persistent symptoms may result from an immune-mediated disruption to the autonomic nervous system. ${ }^{10,11}$ Similar to other post-viral autoimmune conditions (such as Guillain-Barré syndrome), COVID19 infection may act as a trigger for an immune response that causes widespread and debilitating symptoms. ${ }^{1-13}$ These symptoms also overlap with other post-viral autonomic dysfunction syndromes, such as post-viral orthostatic hypotension ${ }^{14}$ and postural orthostatic tachycardia syndrome (POTS). ${ }^{15}$ Because there is no clear pathophysiological pathway for PACS, development of therapeutic agents and rehabilitation protocols has been challenging. 
Due to the highly debilitating nature and extreme lability of PACS symptoms, we developed a novel rehabilitation program known as Autonomic Conditioning Therapy (ACT) for PACS, which is an adaptation of well-established rehabilitative protocols for managing autonomic dysregulation. ${ }^{16,17} \mathrm{At}$ present, there is controversy surrounding the role of exercise programs for this condition, due to concerns over the potential to worsen fatigue. This report compares preliminary longitudinal patient-reported outcome (PRO) data from individuals who completed ACT for PACS, with data from those who did not participate.

This was a retrospective cohort analysis. Participants provided consent for the publication of data and approval was provided by the Mount Sinai Program for Protection of Human Subjects (IRB 20-01985). Inclusion criteria were individuals referred to the Mount Sinai PACS Clinic, with confirmed (by PCR or antibody test) or probable (diagnosed by a medical doctor in accordance with World Health Organization [WHO] recommendations) ${ }^{18}$ prior diagnosis of COVID-19; diagnosed with PACS by a physician using NICE guidelines; ${ }^{5}$ and, completed at least 21 days of ACT for PACS (if enrolled). Exclusion criteria were incomplete data (length of follow-up, fatigue).

Baseline demographic data was collected from individuals' electronic medical records. PRO data were collected with REDCap (Research Electronic Data Capture) electronic data capture tools hosted at Mount Sinai Health System. A REDCap survey link was emailed to individuals. Baseline PRO data were collected from each participant upon enrollment into the PACS clinic as part of standard clinical care, starting in May 2020. This baseline set of PROs included a fatigue visual analogue scale (VAS), Medical Research Council [MRC] Breathlessness Scale, EuroQol health-related quality of life (EQ-5D-5L), Pain VAS, General Anxiety Disorder-7 (GAD-7) and WHO Disability Assessment Schedule (WHODAS). On March 15th 2021, the same PROs were repeated in all individuals, with the addition of the Patient Global Impression of Change (PGIC).

Following initial evaluation in the PACS clinic and cardiac clearance, it was recommended that individuals commence ACT for PACS. Individuals enrolled in ACT for PACS following the completion of 4 weeks of breath work coaching. Breath work coaching focused on ventilatory control and nasal breathing, and was conducted by Stasis (Virginia Beach, VA). ACT for PACS involved participating in 30-minute sessions twice per week physical therapist. This supervised program contained three distinct phases of rehabilitation beginning with supine active range of motion exercises, followed by progressive submaximal aerobic exercises. ${ }^{17}$ Submaximal exercise was monitored using the modified Borg Rate of Perceived Exertion (RPE), and individuals progressed through the phases according to RPE and symptom responses at the therapist's discretion (See supplementary material). Since inception, the ACT for PACS program has been implemented across 53 physical therapy centers in the greater New York area, which all completed standardized ACT for PACS training that was offered by the Mount Sinai Health System. The program was enacted via telehealth or in-person, according to each patient's preference and activity tolerance. 
Statistical analyses were undertaken with Stata (StataCorp, Stata Statistical Software Release: V.14). Data are presented as frequencies and proportions, mean and standard deviation (SD) or median and range where appropriate. Paired Sample t-tests and Independent samples t-tests were used to examine between within- and between-group differences, presented as mean difference ( $95 \%$ confidence interval [CI]). Mann-Whitney U tests were used for between-group comparisons of PGIC and MRC.

Of 94 individuals, 84 (89\%) met the inclusion criteria with complete data. Of these 84,78 (93\%) consented to have their data included in the analyses (Table 1). Three (4\%) could not be reached and three declined to consent. Of the 78 who consented, 31 (40\%) completed ACT for PACS (attending 5 of the trained centers) with the remaining $47(60 \%)$ failing to enroll in the ACT for PACS program due to delays in administrative follow-up and/or referral to physical therapy, or by their own choice. For those in the ACT for PACS group, the median (range) time of participation was 64 (21 to 137) days. 
Table 1

Patient $(n=78)$ baseline demographic and COVID-19 related data.

\begin{tabular}{|c|c|c|c|}
\hline & $\begin{array}{l}\text { All patients ( } \mathrm{n} \\
=78 \text { ) }\end{array}$ & $\begin{array}{l}\text { ACT for PACS ( } \\
=31)\end{array}$ & $\begin{array}{l}\text { No ACT for PACS ( } \\
=47)\end{array}$ \\
\hline Female & $55(71)$ & $22(71)$ & $33(70)$ \\
\hline Age y, median (range) & 43 (12 to 78$)$ & 42 (12 to 73$)$ & 43 (23 to 78$)$ \\
\hline BMI kg/m², median (range) & 24 (16 to 52$)$ & 24 (17 to 34$)$ & 24 (16 to 52$)$ \\
\hline \multicolumn{4}{|l|}{ Race } \\
\hline White & $69(88)$ & $26(84)$ & $43(91)$ \\
\hline Asian & $4(5)$ & $2(6)$ & $2(4)$ \\
\hline Black or African American & $2(3)$ & $1(3)$ & $1(2)$ \\
\hline American Indian or American Native & $1(1)$ & $0(0)$ & $1(2)$ \\
\hline Native Hawaiian or Pacific Islander & $0(0)$ & $0(0)$ & $0(0)$ \\
\hline Other & $4(5)$ & $1(3)$ & $3(6)$ \\
\hline Hispanic or Latinx & $6(8)$ & $2(6)$ & $4(9)$ \\
\hline $\begin{array}{l}\text { Time at follow up in days, median } \\
\text { (range) }\end{array}$ & $\begin{array}{l}186(26 \text { to } \\
238)\end{array}$ & 174 (33 to 237 ) & 194 (26 to 238$)$ \\
\hline PCR completed & $49(63)$ & $21(68)$ & $28(60)$ \\
\hline PCR positive & $13(17)$ & $9(29)$ & $4(9)$ \\
\hline Antibody test completed & $75(96)$ & $30(97)$ & $45(96)$ \\
\hline Antibody positive & $35(45)$ & $15(48)$ & $20(43)$ \\
\hline PCR and/or antibody positive & $39(50)$ & $17(55)$ & $22(47)$ \\
\hline Hospitalized for COVID-19 & $3(4)$ & $3(10)$ & $0(0)$ \\
\hline $\begin{array}{l}\text { Duration of symptoms in days, } \\
\text { median (range) }\end{array}$ & $\begin{array}{l}344 \text { (82 to } \\
457)\end{array}$ & 350 (203 to 457) & 341 (82 to 398 ) \\
\hline \multicolumn{4}{|l|}{ Most common PACS symptoms } \\
\hline Fatigue & $64(82)$ & $26(84)$ & $38(81)$ \\
\hline Headache & $50(64)$ & $18(58)$ & $32(68)$ \\
\hline Difficulty concentrating & $47(60)$ & $16(52)$ & $31(66)$ \\
\hline Dizziness/light-headedness & $45(58)$ & $15(48)$ & $30(64)$ \\
\hline
\end{tabular}




\begin{tabular}{|c|c|c|c|}
\hline & $\begin{array}{l}\text { All patients ( } \mathrm{n} \\
=78 \text { ) }\end{array}$ & $\begin{array}{l}\text { ACT for PACS ( } \\
=31 \text { ) }\end{array}$ & $\begin{array}{l}\text { No ACT for PACS ( } \\
=47)\end{array}$ \\
\hline Difficulty sleeping & $45(58)$ & $18(58)$ & $27(57)$ \\
\hline Heart palpitations & $39(50)$ & $13(42)$ & $26(55)$ \\
\hline Shortness of breath & $36(46)$ & $14(45)$ & $22(47)$ \\
\hline Memory loss & $36(46)$ & $12(39)$ & $24(51)$ \\
\hline \multicolumn{4}{|c|}{ Most prevalent co-morbidities } \\
\hline Cancer (any type) & $22(28)$ & $9(29)$ & $13(28)$ \\
\hline Asthma & $19(24)$ & $6(19)$ & $13(28)$ \\
\hline Anxiety & $10(13)$ & $6(19)$ & $4(9)$ \\
\hline Hypertension & $9(12)$ & $4(13)$ & $5(11)$ \\
\hline Depression & $6(8)$ & $3(10)$ & $3(6)$ \\
\hline
\end{tabular}

Individuals who completed ACT for PACS demonstrated a within-group improvement in fatigue $(p=0.03)$ although this was not observed between groups $(p=0.15)$ (Table 2). Self-reported PGIC was greater in the ACT for PACS group (ACT for PACS median [range] 5 [1 to 7], no ACT for PACS 4 [1 to 7], p< 0.01). In subgroup analyses according to COVID-19 test status by PCR and/or antibody test, individuals with a confirmed positive result demonstrated greater improvement in PGIC (ACT for PACS 6 [1 to 7], no ACT for PACS 3 [1 to 6], $p<0.01$ ), however this was not observed in individuals with a negative/unknown result (ACT for PACS 5 [ 1 to 7], no ACT for PACS 5 [1 to 7], $p=0.62$ ) 
Table 2

Within-group and between group fatigue VAS data for individuals $(n=78)$ who completed ACT for PACS $(n=31)$ and those who did not $(n=47)$, including subgroups of individuals with either positive $(n=39)$ or negative/unknown COVID-19 ( $n=39)$ confirmed by PCR and/or antibody tests.

\begin{tabular}{|c|c|c|c|c|c|c|c|}
\hline \multirow[b]{2}{*}{ Fatigue VAS } & \multicolumn{3}{|c|}{ ACT for PACS } & \multicolumn{3}{|c|}{ No ACT for PACS } & \multirow{2}{*}{$\begin{array}{l}\begin{array}{l}\text { Between } \\
\text { groups }\end{array} \\
\text { Diff. } \\
(95 \% \mathrm{Cl})\end{array}$} \\
\hline & Baseline & $\begin{array}{l}\text { Follow- } \\
\text { up }\end{array}$ & $\begin{array}{l}\text { Diff. } \\
(95 \% \mathrm{Cl})\end{array}$ & Baseline & $\begin{array}{l}\text { Follow- } \\
\text { up }\end{array}$ & $\begin{array}{l}\text { Diff. } \\
(95 \% \\
\text { Cl) }\end{array}$ & \\
\hline All individuals ${ }^{a}$ & $51(20)$ & $36(22)$ & $\begin{array}{l}-14(-27 \\
\text { to }-1)\end{array}$ & $49(27)$ & $48(30)$ & $\begin{array}{l}-1(-13 \\
\text { to } 11)\end{array}$ & $\begin{array}{l}-13(-30 \\
\text { to } 5)\end{array}$ \\
\hline COVID positive ${ }^{b}$ & $50(20)$ & $31(20)$ & $\begin{array}{l}-19(-36 \\
\text { to } 2)\end{array}$ & $48(25)$ & $54(33)$ & $\begin{array}{l}6(-16 \\
\text { to } 26)\end{array}$ & $\begin{array}{l}-25(-52 \\
\text { to } 3)\end{array}$ \\
\hline $\begin{array}{l}\text { COVID } \\
\text { negative/unknown }{ }^{c}\end{array}$ & $51(21)$ & $43(24)$ & $\begin{array}{l}-8(-28 \text { to } \\
13)\end{array}$ & $50(29)$ & $43(26)$ & $\begin{array}{l}-7(-20 \\
\text { to } 7)\end{array}$ & $\begin{array}{l}-1(-24 \text { to } \\
22)\end{array}$ \\
\hline \multicolumn{8}{|c|}{$\begin{array}{l}\text { Data are presented as mean (SD) unless stated otherwise. Analyses performed using Paired and } \\
\text { Independent Samples T tests. VAS = visual analogue scale, ACT = autonomic conditioning therapy, } \\
\text { PACS = post-acute COVID-19 syndrome, Diff. = difference, CI = confidence interval. }\end{array}$} \\
\hline \multicolumn{8}{|c|}{$\begin{array}{l}\text { a ACT for PACS } n=31 \text {, no ACT for PACS } n=47 ;{ }^{b} \text { ACT for PACS } n=17 \text {, no ACT for PACS } n=22 ;{ }^{c} \text { ACT } \\
\text { for PACS } n=14 \text {, no ACT for PACS } n=25 \text {. }\end{array}$} \\
\hline
\end{tabular}

There were no within- or between-group differences observed in other outcomes.

This is the first intervention reported to successfully demonstrate a reduction in fatigue, the most common and debilitating symptom, in individuals with PACS. These improvements were reflected in the greater global impression of change in health reported by individuals when compared with a group who did not participate in the ACT program. Quantifying outcomes in a novel condition with a diverse set of debilitating symptoms can be difficult, and the use of PRO data was considered the best methodology for PACS. The most common symptoms reported in this cohort were fatigue, headache, difficulty concentrating, dizziness/lightheadedness, memory loss, shortness of breath and palpitations.

Developing rehabilitation-based treatments for PACS is uniquely challenging, as most individuals are unable to tolerate traditional exercise and symptoms are often exacerbated with activity. Similar disease features are seen in disorders of the autonomic nervous system, including POTS. ACT for PACS was developed to address these rehabilitation constraints, using principles of graded submaximal aerobic exercise which are known to improve autonomic dysregulation and subsequent fatigue in POTS. ${ }^{17}$ One distinctive feature of ACT for PACS is that individuals progress according to symptom responses rather than elapsed time, resulting in slowly paced yet sustained recovery. Further prospective studies are needed to determine the optimal dose, mode and length of rehabilitation, level of supervision required, and to provide further insights into the characteristics of responders. 
This study has limitations inherent to its non-randomized, retrospective design. Individuals who completed ACT for PACS may have self-selected for efficacy and improvement compared to those who did not complete the program. However, both groups had similar characteristics at baseline, indicating that symptom burden was not a determinant of ACT engagement. Out of caution, and to avoid creating disparities in care, ACT for PACS was deliberately inclusive of individuals diagnosed with COVID-19 by historical/clinical WHO criteria, given the known low sensitivity of PCR and antibody testing ${ }^{19,20}$ and limited test accessibility in New York City early in the pandemic. Since PCR tests became more accessible in the latter half of 2020 and antibody levels can wane quickly, ${ }^{21,22}$ the subgroup with positive PCR and/or antibody tests may actually represent a cohort with a shorter duration of PACS illness, suggesting that ACT should be initiated as early as possible to maximize success. While the ACT for PACS program improved fatigue and PGIC, similar changes were not observed in other outcomes. Further work is required to examine the program under prospective scientific conditions, to determine the impact on a wider range of outcomes that can measure the burden of this novel disease.

ACT for PACS is a physical therapy program utilizing progressive exercise tailored to symptom response that reduces fatigue. Despite early concerns that exercise programs might worsen symptoms, the data reported demonstrate that when appropriately prescribed and implemented, a supervised, gradual, symptom-oriented physical therapy program is well tolerated and should be considered as first line rehabilitation for individuals with PACS.

\section{Declarations}

Acknowledgements

The authors would like to acknowledge the PACS patients who participated in this research, enabling the data for this manuscript, and the frontline healthcare workers working throughout the pandemic. The authors are grateful to the RTW Foundation and the Charles Lazarus Children's Abilities Center for their philanthropic support.

Author Contributions

LT, JTM, ARK, DM, JH and DP conceptualized ACT for PACS and the clinical data collection; JTM coordinated clinical procedures; LT, JTM and JW coordinated data collection. LT, JTM, ARK, JW and DP performed data analysis, wrote, and edited the first manuscript draft; MC, DM, JH, EB, LN, AN, JD, AB and CK provided clinical support and coordination; All authors read and approved the final version of the manuscript.

Competing Interests statement

There was no direct funding received to support this research. There are no conflicts or disclosures to report. 


\section{References}

1. Huang $C$, Huang L, Wang $Y$, et al. 6-month consequences of COVID-19 in patients discharged from hospital: a cohort study. The Lancet. 2021;397(10270):220-232. doi:10.1016/S01406736(20)32656-8

2. Vaes AW, Machado FVC, Meys R, et al. Care Dependency in Non-Hospitalized Patients with COVID-19. J Clin Med. 2020;9(9):2946. doi:10.3390/jcm9092946

3. Tabacof L, Tosto-Mancuso J, Wood J, et al. Post-acute COVID-19 syndrome negatively impacts health and wellbeing despite less severe acute infection. medRxiv. Published online November 6 , 2020:2020.11.04.20226126. doi:10.1101/2020.11.04.20226126

4. Carvalho-Schneider C, Laurent E, Lemaignen A, et al. Follow-up of adults with noncritical COVID-19 two months after symptom onset. Clin Microbiol Infect. Published online October 5, 2020. doi:10.1016/j.cmi.2020.09.052

5. Common symptoms of ongoing symptomatic COVID-19 and post-COVID-19 syndrome | COVID-19 rapid guideline: managing the long-term effects of COVID-19 | Guidance | NICE. Accessed April 6, 2021. https://www.nice.org.uk/guidance/ng188/chapter/common-symptoms-of-ongoingsymptomatic-covid-19-and-post-covid-19-syndrome\#common-symptoms-of-ongoing-symptomaticcovid-19-and-post-covid-19-syndrome

6. Goërtz YMJ, Herck MV, Delbressine JM, et al. Persistent symptoms 3 months after a SARS-CoV-2 infection: the post-COVID-19 syndrome? ERJ Open Res. 2020;6(4). doi:10.1183/23120541.005422020

7. Tenforde MW, Kim SS, Lindsell CJ, et al. Symptom Duration and Risk Factors for Delayed Return to Usual Health Among Outpatients with COVID-19 in a Multistate Health Care Systems Network United States, March-June 2020. MMWR Morb Mortal Wkly Rep. 2020;69(30):993-998. doi:10.15585/mmwr.mm6930e1

8. Greenhalgh T, Knight M, A'Court C, Buxton M, Husain L. Management of post-acute covid-19 in primary care. BMJ. 2020;370:m3026. doi:10.1136/bmj.m3026

9. CDC. COVID Data Tracker. Centers for Disease Control and Prevention. Published March 28, 2020. Accessed January 31, 2021. https://covid.cdc.gov/covid-data-tracker

10. Dani M, Dirksen A, Taraborrelli P, et al. Autonomic dysfunction in 'long COVID': rationale, physiology and management strategies. Clin Med. 2021;21(1):e63-e67. doi:10.7861/clinmed.2020-0896

11. Goldstein DS. The extended autonomic system, dyshomeostasis, and COVID-19. Clin Auton Res. 2020;30(4):299-315. doi:10.1007/s10286-020-00714-0

12. Davido B, Seang S, Tubiana R, de Truchis P. Post-COVID-19 chronic symptoms: a postinfectious entity? Clin Microbiol Infect. 2020;26(11):1448-1449. doi:10.1016/j.cmi.2020.07.028

13. Fudim M, Qadri YJ, Ghadimi K, et al. Implications for Neuromodulation Therapy to Control Inflammation and Related Organ Dysfunction in COVID-19. J Cardiovasc Trans/ Res. 2020;13(6):894-899. doi:10.1007/s12265-020-10031-6 
14. Ricci F, De Caterina R, Fedorowski A. Orthostatic Hypotension: Epidemiology, Prognosis, and Treatment. J Am Coll Cardiol. 2015;66(7):848-860. doi:10.1016/j.jacc.2015.06.1084

15. Bryarly M, Phillips LT, Fu Q, Vernino S, Levine BD. Postural Orthostatic Tachycardia Syndrome: JACC Focus Seminar. J Am Coll Cardiol. 2019;73(10):1207-1228. doi:10.1016/j.jacc.2018.11.059

16. Joyner MJ, Masuki S. POTS versus deconditioning: the same or different? Clin Auton Res. 2008;18(6):300-307. doi:10.1007/s10286-008-0487-7

17. Fu Q, Levine BD. Exercise and non-pharmacological treatment of POTS. Auton Neurosci Basic Clin. 2018;215:20-27. doi:10.1016/j.autneu.2018.07.001

18. WHO COVID-19 Case definition. Accessed April 19, 2021. https://www.who.int/publications-detailredirect/WHO-2019-nCoV-Surveillance_Case_Definition-2020.2

19. Arevalo-Rodriguez I, Buitrago-Garcia D, Simancas-Racines D, et al. False-negative results of initial RTPCR assays for COVID-19: A systematic review. PLOS ONE. 2020;15(12):e0242958. doi:10.1371/journal.pone.0242958

20. Abbasi J. The Promise and Peril of Antibody Testing for COVID-19. JAMA. 2020;323(19):1881-1883. doi:10.1001/jama.2020.6170

21. Long Q-X, Tang X-J, Shi Q-L, et al. Clinical and immunological assessment of asymptomatic SARSCoV-2 infections. Nat Med. 2020;26(8):1200-1204. doi:10.1038/s41591-020-0965-6

22. Ibarrondo FJ, Fulcher JA, Goodman-Meza D, et al. Rapid Decay of Anti-SARS-CoV-2 Antibodies in Persons with Mild Covid-19. N Engl J Med. 2020;383(11):1085-1087. doi:10.1056/NEJMc2025179

\section{Supplementary Files}

This is a list of supplementary files associated with this preprint. Click to download.

- TabacofSupplementaryMaterial.pdf 